International Journal of Power Electronics and Drive Systems (IJPEDS)

Vol. 12, No. 4, December 2021, pp. 2423 2434

ISSN: 2088-8694, DOI: 10.11591/ijpeds.v12.i4.pp2423-2434

2423

\title{
Characterizing power transformer frequency responses using bipolar pseudo-random current impulses
}

\author{
Fredrick Mwaniki ${ }^{1}$, Ahmed A. Sayyid ${ }^{2}$ \\ ${ }^{1}$ Department of Electrical and Electronic Engineering, University of Stellenbosch, Stellenbosch, South Africa \\ ${ }^{2}$ Department of Electrical and Information Engineering, University of Nairobi, Nairobi, Kenya
}

\begin{tabular}{|c|c|}
\hline Article Info & ABSTRACT \\
\hline Article history: & \multirow{11}{*}{$\begin{array}{l}\text { The behaviour of a power transformer is complex and difficult to predict during tran- } \\
\text { sient conditions or during operation at frequencies below or above its nominal fre- } \\
\text { quency, a phenomenon common in renewable energy plants due to harmonic distor- } \\
\text { tion. Furthermore, the accuracy of a power system simulation depends on the models } \\
\text { of critical subsystems such as the power transformers. This paper presents the use of } \\
\text { a unique excitation waveform comprising of pseudo-random current impulses to ac- } \\
\text { curately identify the wideband characteristics of a power transformer. By injecting } \\
\text { the excitation waveform to the relevant transformer terminals, frequency responses are } \\
\text { determined by cross-correlation of the perturbation signal, and the measured response. } \\
\text { Compared to the traditional transformer identification methods, the pseudo-random } \\
\text { current impulses offer a wideband excitation with a higher degree of controllability } \\
\text { such that its spectral energy can be focused in the frequency band of interest. The pro- } \\
\text { posed method was investigated on a } 16 \mathrm{kVA}, 22 \mathrm{kV} / 240 \mathrm{~V} \text { single-phase transformer. } \\
\text { The obtained wideband frequency responses provide useful information in harmonic } \\
\text { penetration and over-voltage studies and are also used to estimate, with a high degree } \\
\text { of accuracy, the lumped parameters of the equivalent transformer broadband circuit } \\
\text { model. }\end{array}$} \\
\hline Received Apr 22, 2021 & \\
\hline ed Sep 23, 2021 & \\
\hline Accepted Sep 27, 2021 & \\
\hline Keywords: & \\
\hline Excitation signals & \\
\hline Frequency response & \\
\hline Harmonics & \\
\hline Parameter estimation & \\
\hline Power quality & \\
\hline Power transformer & \\
\hline
\end{tabular}

This is an open access article under the CC BY-SA license.

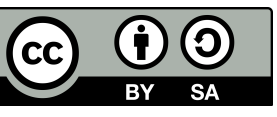

Corresponding Author:

Fredrick Mwaniki

Department of Electrical and Electronic Engineering

Stellenbosch University

Banghoek Road, Stellenbosch, 7600, South Africa

Email: fmmwaniki@sun.ac.za

\section{INTRODUCTION}

In recent years, there has been a rapid increase in renewable energy sources such as photovoltaic and wind generators [1]-[3]. These energy sources have presented new challenges such as the risk of high harmonic voltage distortion due to their use of power electronic converters [1], [4], [5]. These power system harmonics have increased in both amplitude and frequency bandwidth. Step-up transformers that are used to connect these renewable plants to the grid are adversely affected by the harmonics from these renewable plants [6].

Power transformers do not have a flat frequency response, which makes them unsuitable for use in applications with high harmonic components and high frequency transient signals. Transformer impedances are functions of the input signal frequencies and voltage, as well as the loading conditions [7], [8]. Transformer parameters such as leakage inductances, winding capacitances, and the non-linear core components interact to cause complex series and/or parallel resonant phenomena in the transformer frequency characteristics [9]. 
When a minimally damped resonant point in the power transformer's wideband transformation ratio characteristics is excited by a harmonic voltage or a transient event, excessive voltage and/or current amplification occurs. Over-voltages and over-currents may cause protection devices to activate, or cause damage to the power transformer and other equipment connected to the network, resulting to unnecessary downtime.

In this context, the response of transformers to input signals with multiple frequencies needs to be modeled and understood. Therefore, this gives rise to renewed interest in performing broad frequency band measurements, especially on power transformers that are connected to wind and photovoltaic (PV) energy sources. A common approach in transformer identification is the use of non-parametric methods that compute the transformer frequency responses. Frequency response analysis (FRA) has been widely used for condition monitoring of transformers [10]-[15]. Frequency response measurements have also been applied in transformer parameter estimation [10], [16]-[18]. Although power transformer frequency responses have been widely used for condition monitoring and parameter estimation [10]-[12], [18] their use in power quality studies, especially in cases where the transformers are used in renewable energy sources, has not been given much attention.

In practice, frequency response measurements are conducted by applying an excitation signal that can perturb the target system at the frequencies of interest. The system input and output signals are measured over an interval for further processing to obtain the relevant frequency responses. The excitation signals proposed in literature for transformer FRA include the swept sine and the impulse [11], [19], [20]. The sine sweep involves a variable-frequency sinusoidal signal which is simple to implement using low-voltage sinusoidal signal generators. In practice, however, it is difficult to design and implement a sinusoidal source to perturb a large power transformer over the frequency range of interest, especially at low and high ends of the range. This is due to reduced magnetizing reactance and winding capacitive reactance at these frequencies respectively. Consequently, very low signal levels result in measurement errors due to a low signal to noise ratio (SNR). The impulse method involves perturbing the transformer with a classical voltage impulse Which has a low rise time and a higher fall time. Although this method is more accurate, the spectral energy of the classical impulse waveform drops rapidly with frequency which would result to limited accuracy in some frequencies.

Controlled signals have been proposed for use in power system equipment identification [21]-[23], and have advantages in that their spectral characteristics can be controlled and are repeatable. Therefore, accurate measurements are possible at the expense of complex instrumentation [11]. Despite the advantages presented by controlled perturbation signal method, there has been limited application of these signals for transformer FRA. In the case of a pseudorandom binary sequence (PRBS), the clock frequency and sequence length can be controlled to induce persistent excitation for the dynamic modes associated with the device under test. The PRBS has a flat frequency spectrum at frequencies below the $-3 \mathrm{~dB}$ point which allows for perturbation with uniform energy [24]. The PRBS is however not suitable as a perturbation signal for transformers due to a possibility of injecting low frequency components that would cause core saturation. This particularly occurs when a long PRBS is used [25]. The main contribution of this work, therefore, includes the following:

- The use of a wideband controlled perturbation signal to obtain accurate transformer frequency responses.

- The use of transformer frequency responses for power quality studies and parameter estimation.

In this paper the use of a unique signal consisting of bipolar pseudo-random current impulses as a broadband excitation signal to determine the frequency responses of a transformer in the harmonic frequency range is proposed. The obtained responses are then used to study the effects of harmonics especially on the voltage transfer function, to characterise the power transformer over a wide frequency band as well as to obtain transformer wideband parameters. The sequence combines the advantages of the PRBS and the classical double exponential type impulse. An advantage of using this signal is that its power spectrum can be controlled, for instance, in reducing the energy in low frequencies to avoid transformer saturation during measurements. Properties of the signal such as the clock frequency and time constants can be used to focus the spectral energy to the frequency range of interest and improve the SNR. The bipolar nature of the sequence ensures that the transformer is not driven towards a biased offset point from the normal operating point whereas its wideband nature allows tests to be conducted within short time periods compared to conventional methods such as the sine sweep.

In section 2, a methodology for transformer frequency response measurements is discussed. Experimental results and parameter estimation of a high frequency power transformer model using the experimental frequency responses is discussed in section 3. The conclusion is presented in section 4 . 


\section{RESEARCH METHOD}

\subsection{Transformer testing using a sequence of bipolar pseudo-random current impulses}

The idea of using a bipolar pseudo-random impulse sequence as a perturbation signal in the energy systems and power equipment was first proposed in [26]. The bipolar pseudo-random impulse sequence is a wideband signal consisting of a series of chopped positive and negative impulses. In this work, a sequence of bipolar pseudo-random current impulses was used as a perturbation signal for transformer identification and modelling. Figure 1 illustrates the bipolar pseudo-random impulse sequence. Bipolar pseudo-random current impulses can be generated using an efficient circuit topology consisting of a DC source, a full-bridge whose switches are controlled by a PRBS gate signal and a series RLC network as shown in Figure 2.

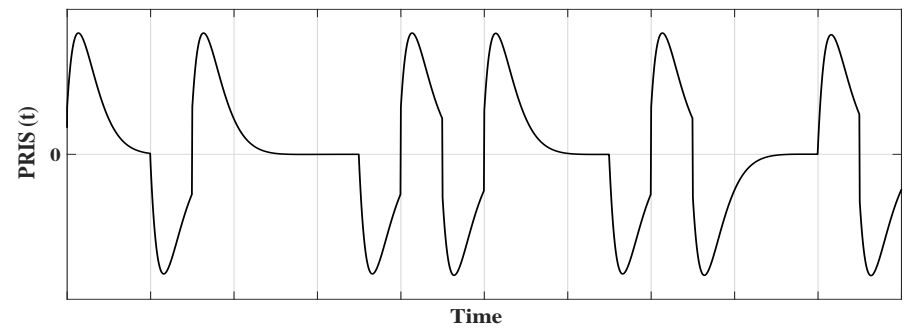

Figure 1. Bipolar pseudo-random impulse sequence

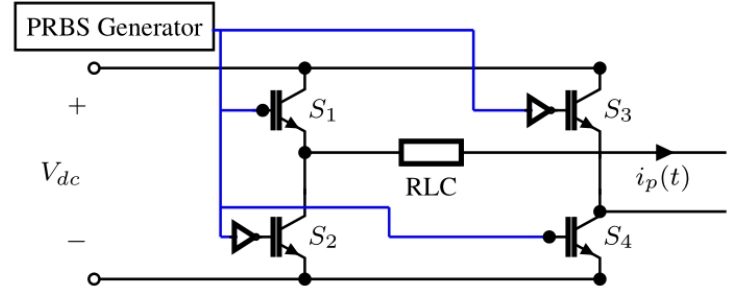

Figure 2. Circuit diagram for the bipolar Pseudo-random current impulses generator

The PRBS is a random, deterministic and periodic signal that can be produced using shift registers driven by a clock sequence. The occurrence and duration of the binary states is random. The data length for one PRBS period is defined as $N=2^{n}-1$ bits, where $n$ denotes the number of shift registers. The series RLC network provides a means of optimizing the time-constants associated with the rise-and fall-times of the bipolar Pseudo-random current impulses. The circuit of Figure 2 can be viewed as an application of a step input to a series RLC circuit, where the step is controlled by the PRBS signal. The sequence of bipolar current impulses generated by the circuit in Figure 2 over one period can be expressed as:

$$
\begin{aligned}
i_{p}(t)= & \sum_{i=0,2,4 \ldots}^{((N+1) / 2)-1} \frac{\left[e^{-t / \tau_{1}}\left(V_{0}-V_{d c}+\frac{I_{0} L}{\tau_{1}}\right)-e^{-t / \tau_{2}}\left(V_{0}-V_{d c}+\frac{I_{0} L}{\tau_{2}}\right)\right] \tau_{1} \tau_{2}}{L\left(\tau_{2}-\tau_{1}\right)} \times\left[u\left(t-t_{i}\right)-u\left(t-t_{i+1}\right)\right] \\
& -\sum_{i=1,3,5 \ldots}^{((N+1) / 2)} \frac{\left[e^{-t / \tau_{1}}\left(V_{0}-V_{d c}+\frac{I_{0} L}{\tau_{1}}\right)-e^{-t / \tau_{2}}\left(V_{0}-V_{d c}+\frac{I_{0} L}{\tau_{2}}\right)\right] \tau_{1} \tau_{2}}{L\left(\tau_{2}-\tau_{1}\right)} \times\left[u\left(t-t_{i}\right)-u\left(t-t_{i+1}\right)\right] .
\end{aligned}
$$

where $u\left(t-t_{i}\right)-u\left(t-t_{i+1}\right)$ depict random switching intervals in the full bridge circuit, $\tau_{1}$ and $\tau_{2}$ are the time constants associated with the series RLC network, $I_{0}$ and $V_{0}$ denote the initial current through the inductor and the initial voltage across the capacitor respectively.

In (1) indicates that the excitation current depends on the DC source, the RLC network time constants and the PRBS switching intervals which in turn are dependent on the PRBS clock frequency. In practice, 
the shape of the perturbation signal is also affected by the initial energy in the inductor and capacitor in each switching cycle. The properties of the current impulses can easily be controlled by varying excitation source parameters namely the input DC source, PRBS clock frequency $f_{c}$, and the impulses time constants. This high degree of control offers capability to improve the signal's SNR, guarantee repeatable experiments and allow the user to concentrate the signal's energy in the required frequency band. The broadband nature of the sequence ensures that the transformer identification procedure is conducted fast compared to using conventional excitation signals such as stepped sine. This is an advantage especially in in situ power system measurements where parameters such as harmonics are time-dependent. The bipolar pseudo-random impulse sequence can, therefore, be adjusted to achieve persistent excitation for a broad range of frequencies and applications. This is an important aspect especially in identification of systems involving electromagnetic components such as transformers where superior performance is achieved when excitation energy is directed in the higher frequency band and the low frequency perturbation is restricted.

\subsection{Transformer test arrangement}

\subsubsection{Offline frequency response measurements}

In offline frequency response measurements, the transformer is disconnected from the normal AC supply voltage. A perturbation source is then connected to the transformer terminals thus providing excitation. Figure 3 shows a test arrangement that can be used to obtain the frequency response of the secondary opencircuit input impedance. This is the impedance seen from the secondary terminals of transformer $T_{r}$ with primary terminals left open. A perturbation source is connected to the secondary terminals as shown in Figure 3. The waveforms of the applied perturbation signal, $i_{p}(t)$, and the perturbed voltage across the secondary winding, $v_{s}(t)$, are then recorded over a time interval.

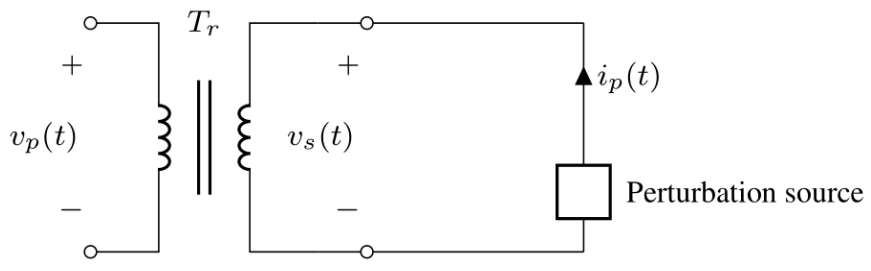

Figure 3. Test arrangement for secondary open-circuit input impedance frequency response

Regarding the transformer as a linear time-invariant system and ignoring measurement and quantization noise, the sampled voltage is given by the discrete convolution of the impulse response with the perturbation signal which can be expressed as.

$$
v_{s}[n]=\sum_{k=1}^{\infty} z[k] i_{p}[n-k]
$$

where $v_{s}(n)$ is the discrete-time voltage signal, $i_{p}(k)$ is the discrete-time perturbation signal and $z(k)$ is the sampled system impulse response.

Convolution of time domain signals implies multiplication of the signals in frequency-domain and therefore, the secondary open-circuit input impedance frequency response $Z_{s}(\omega)$ can be obtained by applying the Fourier transform to the measured voltage and current signals. $Z_{s}(\omega)$ can then be expressed as.

$$
Z_{s}(\omega)=\frac{\mathcal{F}\left\{v_{s}(t)\right\}}{\mathcal{F}\left\{i_{p}(t)\right\}}
$$

where $\mathcal{F}$ denotes the Fourier transform.

The primary open-circuit input impedance frequency response $Z_{p, \text { open }}(\omega)$ can be obtained from the test arrangement shown in Figure 4. The excitation source is connected to the primary winding terminals and the perturbation current, $i_{p}(t)$, and the perturbed voltage across the primary winding, $v_{p}(t)$, are then recorded over a time interval. 


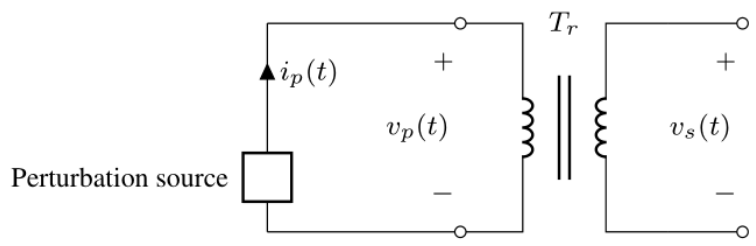

Figure 4. Test arrangement for primary open-circuit input impedance frequency response

The frequency response of the primary open-circuit input impedance can be obtained from the expression

$$
Z_{p_{\text {,open }}}(\omega)=\frac{\mathcal{F}\left\{v_{p}(t)\right\}}{\mathcal{F}\left\{i_{p}(t)\right\}}
$$

The frequency response of the transformation ratio $T_{R}(\omega)$ can be obtained using the topology shown in [4] by recording the perturbed voltages across both primary and secondary windings. The transformation ratio frequency response can be expressed as.

$$
T_{R}(\omega)=\frac{\mathcal{F}\left\{v_{p}(t)\right\}}{\mathcal{F}\left\{v_{s}(t)\right\}}
$$

By short-circuiting the secondary terminals of the test arrangement shown in Figure 4 the primary short-circuit input impedance frequency response can be obtained using the expression in (4).

\subsubsection{In situ frequency response measurements}

In in situ measurements, the transformer stays connected to the conventional supply voltage during perturbation. The transformer does not need to be disconnected, which reduces down time. Furthermore, during in situ measurements, the effects of the normal transformer operation are accounted for [26]. The dynamic range of the excitation signal however has to be kept low enough to ensure that the transformer is not perturbed too far from its optimal operating point.

Figure 5 shows a connection scheme that can be used to characterize, in situ, the secondary opencircuit input impedance $Z_{s}$. The transformer is connected to its normal ac voltage supply $V_{T h}$ which has an internal impedance $Z_{T h}$. The perturbation source is then connected across the voltage source, thereby injecting the excitation current into the transformer terminals. The perturbation current, $i_{p}(t)$, causes a voltage perturbation to be superimposed on the supply voltage $V_{T h}$ due to a voltage drop across $Z_{T h}$.

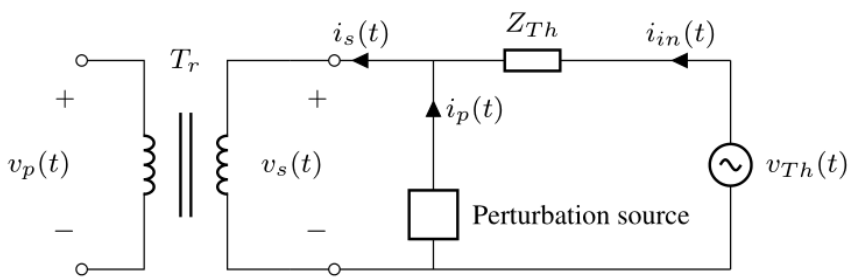

Figure 5. Test connection for in situ transformer perturbation

The perturbed voltage $v_{s}(t)$ and current $i_{s}(t)$ into the transformer are then measured over a time interval. The secondary open-circuit input impedance frequency response $Z_{s}(\omega)$ can be obtained from the expression,

$$
Z_{s}(\omega)=\frac{\mathcal{F}\left\{v_{s}(t)\right\}}{\mathcal{F}\left\{i_{s}(t)\right\}}
$$

A similar arrangement can be used to obtain the voltage transformation ratio whereas the secondary short-circuit input impedance measurement can be done with the primary-side terminals shorted. In in situ measurements, a high SNR can be achieved with lower excitation compared to the offline measurements. 


\section{RESULTS AND ANALYSIS}

\subsection{Frequency response analysis}

Verification of the proposed perturbation method is done by perturbing, offline, a $16 \mathrm{kVA}, 22 \mathrm{kV} / 240$ $\mathrm{V}$ single-phase distribution transformer using a pseudo-random current impulse described in section 2.1. The perturbation signal is generated by controlling the $\mathrm{H}$-bridge switches using a PRBS gate signal with $f_{c}$ set to $15 \mathrm{kHz}$ thereby switching a series RLC network with values $R=50, L=100 \mu H, C=2 \mu F$. A DC voltage of $60 \mathrm{~V}$ is applied to the H-bridge input.

The excitation current generated is then applied to the transformer terminals using the test arrangements discussed in section 2.2 to obtain the various frequency response estimates. Each measurement is conducted for a duration of $1 \mathrm{~s}$ and the required voltage and current data recorded. Voltage and current waveforms are recorded using the GWinstek GDP-025 differential voltage probe and the A622 Tektronix current probe respectively. The National Instruments CompactDAQ chassis and the NI 9223 module is used for data acquisition. Figure 6 shows the measured waveforms for the bipolar Pseudo-random perturbation current and the PRBS gate signal obtained for the circuit shown in Figure 2.

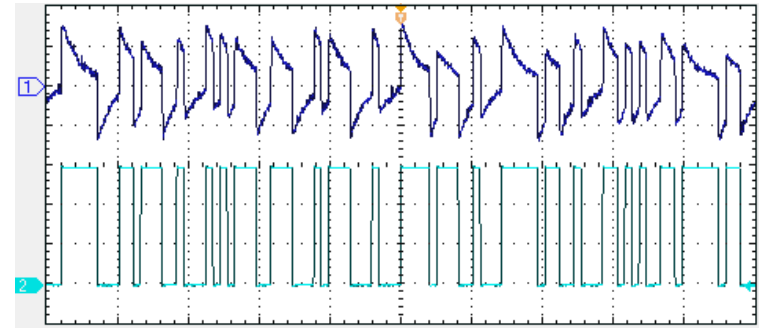

Figure 6. Experimental waveforms for the bipolar Pseudo-random perturbation current (top) and PRBS gate signal (bottom)

To eliminate stochastic measurement noise and reduce leakage and aliasing problems, the acquired data is processed in Matlab using the Welch estimator where the measured data is divided into overlapped and windowed segments. The transfer function of each segment is computed and then averaged to generate a frequency response. The Hann window was selected as it offered good frequency resolution and an overlap percentage of $50 \%$ is used. The low voltage (LV) open circuit input impedance is measured by using the test arrangement shown in Figure 3.

In this case, the bipolar pseudo-random current impulses generator is connected to the LV side terminals of the transformer while the high voltage (HV) winding is left open. The perturbed LV Voltage and the perturbation current are recorded and used for the impedance estimation. The obtained experimental LV open circuit input impedance magnitude and phase frequency responses are shown in Figure 7.

The impedance frequency response in Figure 7 indicates various resonance peaks. The first resonance peak indicates a parallel resonance at approximately $100 \mathrm{~Hz}$, a series resonance at about $6 \mathrm{kHz}$ and a parallel and series resonance pair between $20 \mathrm{kHz}$ and $30 \mathrm{kHz}$. A highly damped parallel and series resonance pair is also noticeable between $40 \mathrm{kHz}$ and $50 \mathrm{kHz}$.

Experiments to determine the transformer input response from the HV side are conducted using the test arrangement shown in Figure 4. To determine the HV short-circuit input impedance, the perturbation current is applied to the HV terminals with the LV terminals shorted. The perturbed HV Voltage and the injected current are recorded and used in the estimation of the impedance response. In this case, a large current is drawn by the winding resistances and leakage inductances due to the shorted LV winding. Figure 8 shows the obtained experimental short-circuit input impedance magnitude and phase frequency responses of the HV side of the transformer. A parallel resonance at about $2.5 \mathrm{kHz}$ and a series resonance at about $3 \mathrm{kHz}$ are evident. At low frequencies, up to about $2 \mathrm{kHz}$, the impedance is predominantly inductive which can be attributed to the leakage inductance of the transformer. However, at higher frequencies beyond $4 \mathrm{kHz}$, the impedance turns capacitive which can be attributed to the large HV winding capacitor.

The transformation ratio is obtained by applying the pseudo-random current impulse sequence to the HV side with the LV winding open. Voltage waveforms $v_{H V}(t)$ and $v_{L V}(t)$ are recorded and used to estimate the transformation ratio frequency response. Figure 9 shows the obtained experimental HV to LV transformation ratio magnitude and phase frequency responses. 

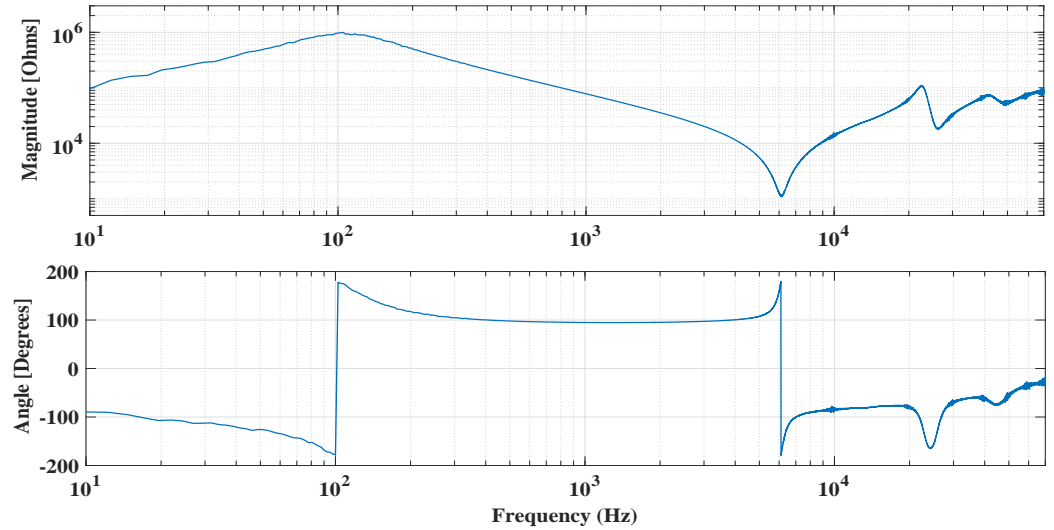

Figure 7. Experimental LV open-circuit input impedance magnitude (top) and phase (bottom) frequency responses
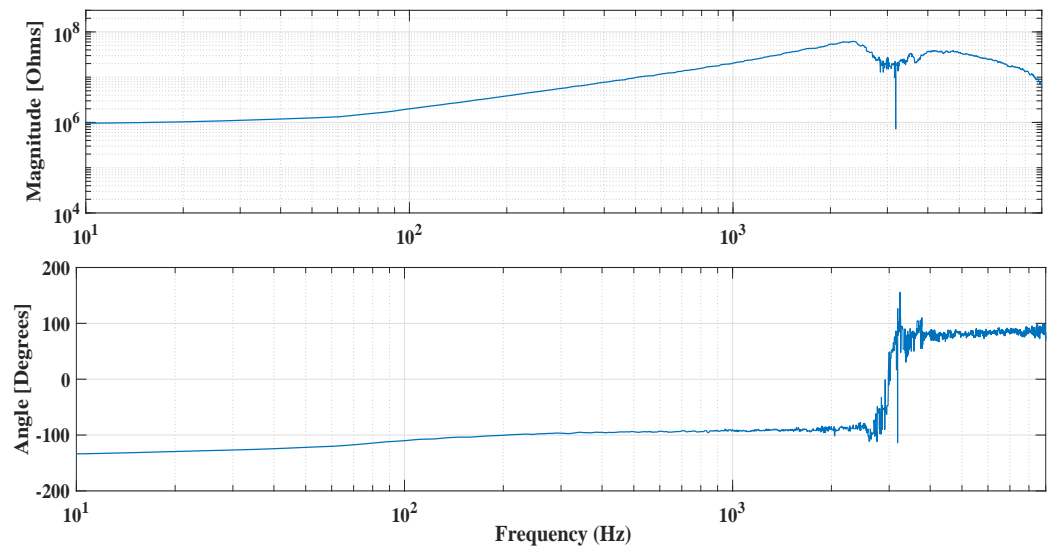

Figure 8. Experimental HV short-circuit input impedance magnitude (top) and phase (bottom) frequency responses
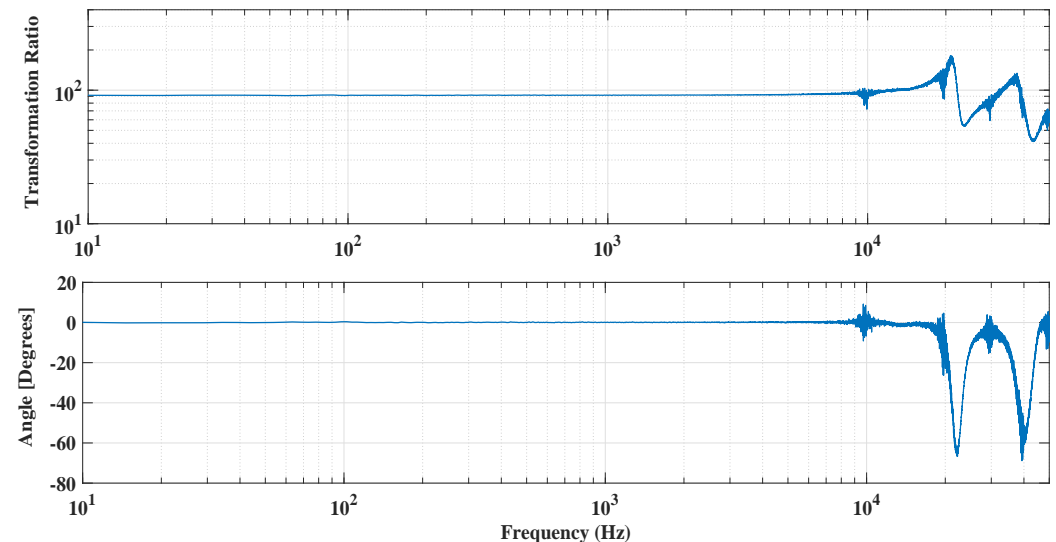

Figure 9. Experimental HV-LV voltage transformation ratio magnitude (top) and phase (bottom) frequency responses

From the magnitude response of the transformation ratio, it can be seen that at low frequencies up to approximately $10 \mathrm{kHz}$, the ratio $V_{H V}(f) / V_{L V}(f)$ is flat at a value of about 91 as expected. As the frequency increases beyond $10 \mathrm{kHz}$, resonant peak pairs are observed to appear at frequencies beyond $20 \mathrm{kHz}$. These 
peaks in the voltage transformation ratio are attributed to the resonance modes caused by the interaction of various transformer elements. It is seen that the voltage transfer at $20 \mathrm{kHz}$ is about twice the voltage transfer at the fundamental frequency of $50 \mathrm{~Hz}$. The presence of resonance characteristics in the wideband transformation ratio such as the one shown in Figure 9 presents challenges to the use of classical distribution transformers in applications that are prone to harmonic distortion and high frequency transient signals such as renewable energy plants.

\subsection{Transformer equivalent model and parameter estimation}

A transformer model with parameters representing its physical behaviour is important for designers [27]. Such a model can also be used for power system simulation. The tested transformer was modelled using the circuit presented in Figure 10 which shows the lumped-parameter equivalent circuit model. The circuit has been simplified by referring the secondary circuit parameters to the primary side of the transformer.

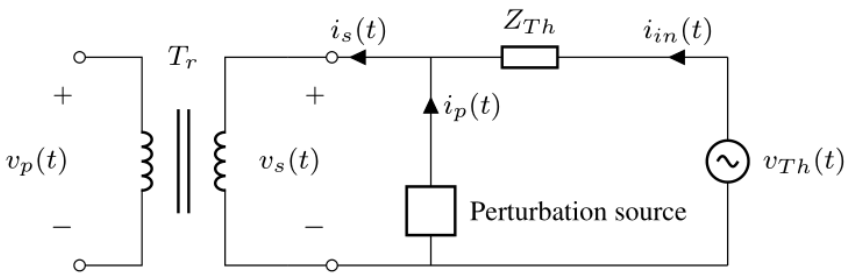

Figure 10. Lumped parameter circuit transformer model

$C_{p}, C_{s}$ and $C_{p s}$ denote the transformer primary winding, secondary winding and inter-winding capacitances respectively. $R_{s}$ and $R_{e}$ denote the transformer winding resistance and core losses resistor respectively while $L_{s}$ and $L_{m}$ denote the leakage inductance and magnetizing inductance respectively. The parameter estimation process is summarised in the flowchart shown in Figure 11.

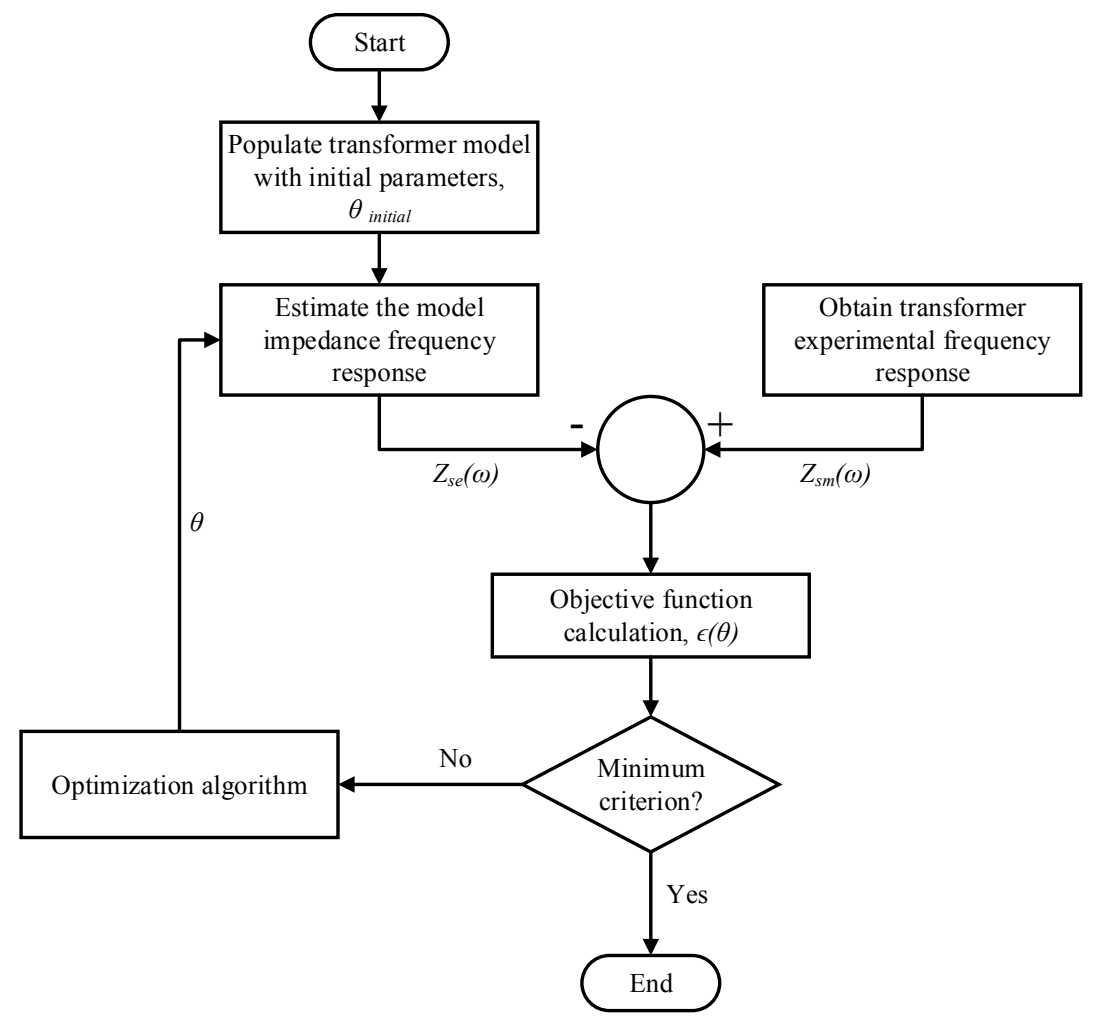

Figure 11. Flowchart of the transformer parameter estimation procedure used in this study 
The current signal, $i_{p}(t)$, that was measured in the LV open circuit input impedance experiment is transformed to the intermediate voltage level using the ratio $\frac{1}{T_{R}}$. The transformed current signal is applied to a Simulink model of the circuit in Figure 10, whose parameters have been initialised with values based on a priori knowledge of the transformer under investigation. The secondary voltage signal at intermediate voltage level is then simulated. The transformed current and the simulated voltage signals are used to estimate the frequency response of the secondary open-circuit input impedance, $Z_{s e}(\omega)$. The measured and estimated responses, $Z_{s m}(\omega)$ and $Z_{s e}(\omega)$, were compared to yield an objective function.

The objective function used in this estimation can be stated as.

$$
\epsilon(\theta)=\sum_{k=1}^{M}\left|Z_{s m}\left(\omega_{k}\right)-Z_{s e}\left(\omega_{k}\right)\right|^{2},
$$

where $M$ indicates the number of discrete frequency points in the compared frequency responses and $\epsilon(\theta)$ is the objective function and $\theta$ is the vector of parameters to be estimated. The considered equivalent circuit of a single-phase power transformer leads to a parameter vector that can be represented as.

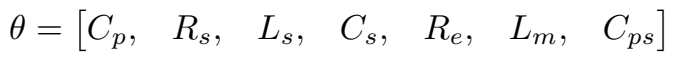

The optimization procedure was used to minimize the objective function by repetitively changing the transformer model parameters, such that the difference between $Z_{s m}(\omega)$ and $Z_{s e}(\omega)$ was at its minimum value. The fmincon optimization procedure was used as the optimization algorithm to reduce the objective function in this investigation. Table 1 presented the estimated parameter values obtained for the test transformer.

Table 1. Estimated parameter values for the transformer model in Figure 10

\begin{tabular}{cccccc}
\hline Parameter & Value & Parameter & Value & Parameter & Value \\
\hline$C_{p}$ & $2 \mathrm{nF}$ & $C_{p s}$ & $0.1375 \mathrm{nF}$ & $C_{s}$ & $31.75 \mathrm{pF}$ \\
$R_{s}$ & $1 \mathrm{k}$ & $R_{e}$ & $1 \mathrm{M}$ & & \\
$L_{s}$ & $0.32 \mathrm{H}$ & $L_{m}$ & $1350 \mathrm{H}$ & & \\
\hline
\end{tabular}

The measured and estimated frequency responses of the LV open-circuit input impedance are shown in Figure 12. It can be seen that the considered transformer model provides a reasonable good prediction of the frequency response up to $20 \mathrm{kHz}$, despite its relative simplicity. The location of the major resonant frequencies of the response, at $100 \mathrm{kHz}$ and $6.2 \mathrm{kHz}$, and the amount of damping associated with these resonances is well predicted. However, the order of the model is too low to accurately predict the minor resonances occurring at higher frequencies. The transformer model can be improved by taking into account the non-linear phenomena such as magnetic saturation and frequency-dependence of the winding resistances due to skin effect. The inductance of the measurement cables between the perturbation source and the transformer terminals, which resonates with the transformer stray capacitors, also need to be taken into account in the model.

Frequencies at which the resonance occurs can be confirmed using (9)

$$
f_{r}=\frac{1}{2 \pi \sqrt{L C}}
$$

where $f_{r}$ is the resonant frequency, $L$ and $C$ denote the inductance and capacitance respectively.

For instance, the first parallel resonance in Figure 12 occurs when $L_{m}$ resonates with $C_{p}$ while the first series resonance can be attributed to $L_{s}$ resonating with $C_{p}$. To validate the obtained parameters, the transformer model shown in Figure 10 was populated with the parameters in Table 1 and simulated by perturbing it using a sine sweep. The model was used to estimate the frequency response of the transformation ratio. Figure 13 shows that the simulated results match with the measured results in Figure 9 indicating resonance points between $20-30 \mathrm{kHz}$. 


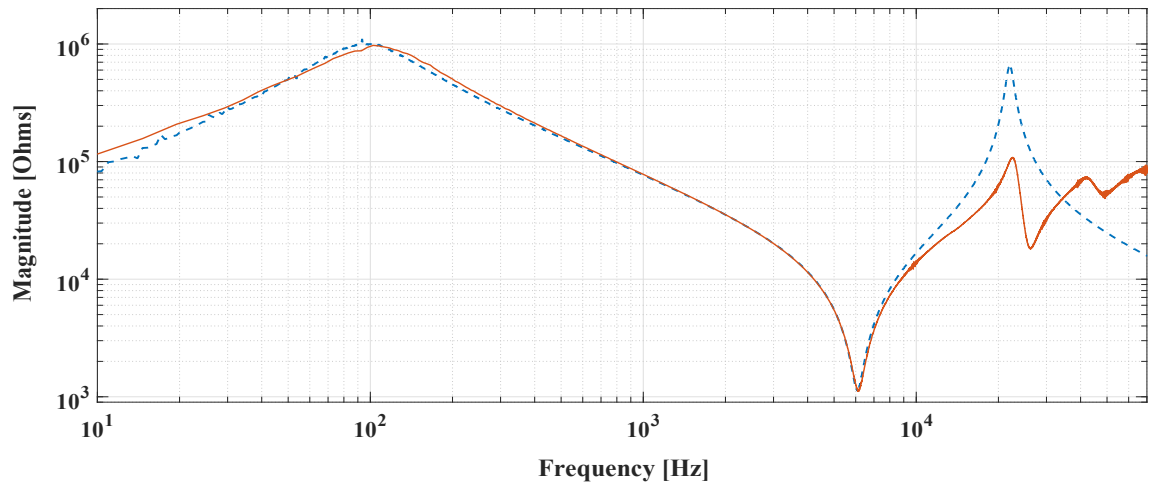

Figure 12. Measured and estimated frequency responses of the LV open-circuit input impedance; 1) solid-measured, 2) dashed-estimated

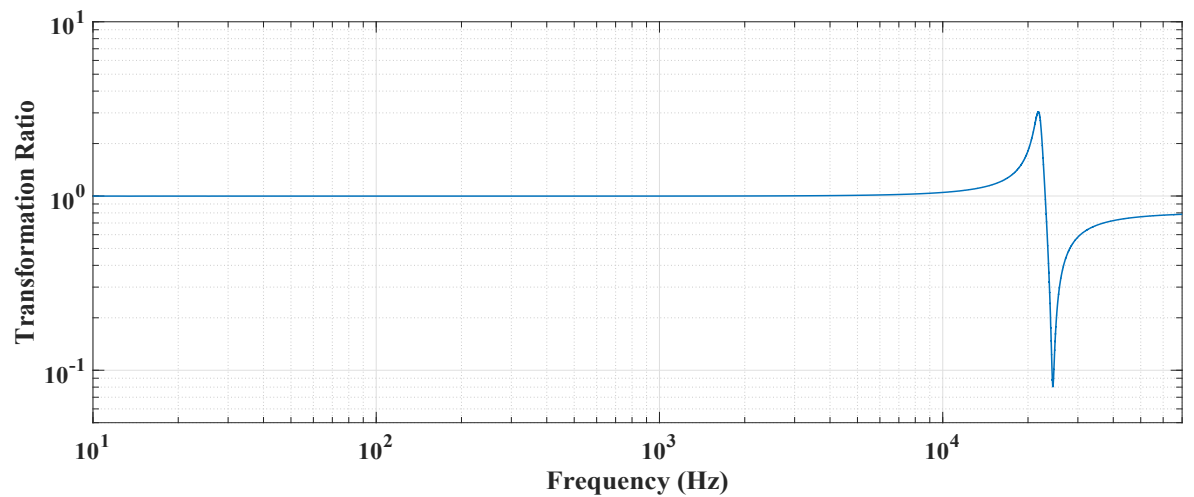

Figure 13. Transformation ratio frequency response simulated using the estimated parameters

\section{CONCLUSION}

A novel methodology that can be used to study the behaviour of power transformers not only at the fundamental frequency but also at harmonic frequencies is presented. Accurate frequency responses are obtained by perturbing a $16 \mathrm{kVA}, 22 \mathrm{kV} / 240 \mathrm{~V}$ power transformer using a sequence of bipolar pseudo-random current impulses. The time-and frequency-domain properties of this wideband excitation signal are controllable which makes it particularly suitable in transformer identification where excitation energy should be concentrated towards the higher frequency band with limited low frequency components. This reduces the saturation effects of the electromagnetic system and is a major advantage of the proposed perturbation signal for transformer identification, compared to other approaches such as those utilising sine sweep, impulse and PRBS. The proposed method can be used to study the impact of harmonics especially on transformers connected to renewable energy sources. Furthermore, the measured frequency response results can be used to estimate the parameter values of a wideband lumped parameter equivalent circuit model of the transformer. As future work, the accuracy of the estimated wideband transformer parameters can be improved by considering non-linear phenomena such as core saturation and skin effect. The non-linearity will result in additional model parameters and hence a higher order transformer model.

\section{REFERENCES}

[1] R. Murray and J. A. de Kock, "A proposed method for evaluating the frequency response of $22 \mathrm{kV}$ outdoor current transformer for harmonic measurements in renewable energy plant applications," 2019 IEEE 10th International Workshop on Applied Measurements for Power Systems (AMPS), 2019, pp. 1-6, doi: 10.1109/AMPS.2019.8897784.

[2] S. N. Nikolovski, P. Z. Maric, and L. V. Majdandzic, "Integration of Solar Power Plant in Distribution Network," International Journal of Electrical and Computer Engineering (IJECE), vol. 5, no. 4, pp. 656-668, Aug. 2015. 
[3] E. Kabalci, "Design and analysis of a hybrid renewable energy plant with solar and wind power," Energy Conversion and Management, vol. 72, pp. 51-59, Aug. 2013, doi: 10.1016/j.enconman.2012.08.027.

[4] G. Reina, M. Mauledoux, and O. A. Aviles, "Simulation of a microgrid for a non-interconnected zone that integrates renewable energies," International Journal of Electrical and Computer Engineering (IJECE), vol. 11, no. 1, pp. 201-216, Feb. 2021, doi: 10.11591/ijece.v11i1.pp201-216.

[5] J. M. Villanueva-Ramirez, P. Gomez, and R. T. Meyer, "Optimized dielectric design of transformer windings under fast-front voltage pulses from power electronic converters," International Journal of Electrical Power and Energy Systems, vol. 129, p. 106849, Jul. 2021, doi: 10.1016/j.ijepes.2021.106849.

[6] S. Ozdemir, N. Altin, and I. Sefa, "Single stage three level grid interactive MPPT inverter for PV system," Energy Conversion and Management, vol. 80, pp. 561-572, Apr. 2014, doi: 10.1016/j.enconman.2014.01.048.

[7] A. N. Hamoodi, B. A. Hamaad, and F. S. Abdullah, "Experimental simulation analysis for single phase trasformer tests," Bulletin of Electrical Engineering and Informatics, vol. 9, no. 3, pp. 862-869, Jun. 2020, doi: 10.11591/eei.v9i3.1710.

[8] A. Keyhani, S. M. Miri, and S. Hao, "Parameter estimation for power transformer models from time-domain data," IEEE Transactions on Power Delivery, vol. 1, no. 3, pp. 140-146, Jul. 1986, doi: 10.1109/TPWRD.1986.4307985.

[9] B. Gustavsen, "Wide band modeling of power transformers," in IEEE Transactions on Power Delivery, vol. 19, no. 1, pp. 414-422, Jan. 2004, doi: 10.1109/TPWRD.2003.820197.

[10] X. Zhao, C. Yao, A. Abu-Siada, and R. Liao, "High frequency electric circuit modeling for transformer frequency response analysis studies," Electrical Power and Energy Systems, vol. 111, pp. 351-368, Oct. 2019, doi: 10.1016/j.ijepes.2019.04.010.

[11] E. Gomez-Luna, G. Aponte Mayor, C. Gonzalez-Garcia, and J. Pleite Guerra, "Current status and future trends in frequency-response analysis with transformer in service," in IEEE Transactions on Power Delivery, vol. 28, no. 2, pp. 1024-1031, Apr. 2013, doi: 10.1109/TPWRD.2012.2234141.

[12] M. K. Ilampoornan and M. Vikash, "Transformer fault detection by frequency response analysis," IOSR Journal of Electrical and Electronic Engineering, vol. 1, no. 4, pp. 27-32, July- August 2012, doi: 10.9790/1676-0142732.

[13] P. Mraz, P. Treyer, U. Hammer, and S. Gonzalez, "Innovative application of frequency response analysis for partial discharge measurement," in 19th International Symposium on High Voltage Engineering, vol. 1, August 2015.

[14] M. Eslamian, B. Vahidi, and S. H. Hosseinian, "Analytical calculation of detailed model parameters of cast resin dry-type transformers," Energy Conversion and Management, vol. 52, no. 7, pp. 2565-2574, July 2011, doi: 10.1016/j.enconman.2011.01.011.

[15] R. M. Youssouf, R. S. A. Ferreira, F. Meghnefi, H. Ezzaidi, P. Picher, and I. Fofana, "Frequency response of transformer winding: A case study based on a laboratory model," in 2018 IEEE Conference on Electrical Insulation and Dielectric Phenomena (CEIDP), 2018, pp. 271-274, doi: 10.1109/CEIDP.2018.8544803.

[16] H. J. Vermeulen, L. R. Dann, and J. Vaan Rooijen, "Equivalent circuit modelling of a capacitive voltage transformer for power system harmonic frequencies," in IEEE Transactions on Power Delivery, vol. 10, no. 4, pp. 1743-1749, Oct. 1995, doi: 10.1109/61.473385.

[17] F. Ghassemi, P. Gale, T. Cumming, and C. Coutts, "Harmonic voltage measurements using CVTs," in IEEE Transactions on Power Delivery, vol. 20, no. 1, pp. 443-449, Jan. 2005, doi: 10.1109/TPWRD.2004.837675.

[18] A. A. Siada, M. I. Mosaas, D. Kim, and M. F. El-Naggar, "Estimating power transformer high frequency model parameters using frequency response analysis," in IEEE Transactions on Power Delivery, vol. 35, no. 3, pp. 12671277, Jun. 2020, doi: 10.1109/TPWRD.2019.2938020.

[19] S. Alsuhaibani, Y. Khan, and A. Beroual, "A review of frequency response analysis methods for power transformer diagnosis," Energies, vol. 9, no. 11, p. 879, October 2016, doi: 10.3390/en9110879.

[20] H. Rahimpour, S. Mitchell, and S. Rahimpour, "Online monitoring of power transformers using impulse frequency response analysis," in 2017 Iranian Conference on Electrical Engineering (ICEE), 2017, pp. 1390-1394, doi: 10.1109/IranianCEE.2017.7985259.

[21] R. Hidalgo-Leon, J. Urquizo, J. Litardo, Y. Munoz-Jadan, P. Singh, and J. Wu, "Simulation of battery discharge emulator using power electronics device with cascaded P-I control," 2020 IEEE International Conference on Industrial Technology (ICIT), 2020, pp. 959-964, doi: 10.1109/ICIT45562.2020.9067170.

[22] H. J. Vermeulen and J. M. Strauss, "Off-line identification of an open-loop automatic voltage regulator using pseudorandom binary sequence perturbations," 1999 IEEE Africon. 5th Africon Conference in Africa (Cat. No.99CH36342), 1999, pp. 799-802 vol.2, doi: 10.1109/AFRCON.1999.821870.

[23] H. J. Vermeulen, J. M. Strauss, and V. Shikoana, "Online estimation of synchronous generator parameters using PRBS perturbations," in IEEE Trans. Power Sys., vol. 17, no. 3, pp. 694-700, Aug. 2002, doi: 10.1109/TPWRS.2002.800915.

[24] S. W. Sung and J. H. Lee, "Pseudo-random binary sequence design for finite impulse response identification," Control Engineering Practice, vol. 11, no. 8, pp. 935-947, Aug. 2003, doi: 10.1016/S0967-0661(03)00035-2.

[25] J. Vaan Rooijen and H. J. Vermeulen, "A perturbation source for in-situ parameter estimation applications," Proceedings of IECON'94-20th Annual Conference of IEEE Industrial Electronics, 1994, pp. 1819-1823 vol.3, doi: 
10.1109/IECON.1994.398092.

[26] F. M. Mwaniki and H. J. Vermeulen, "Characterization and application of a pseudorandom impulse sequence for parameter estimation applications," in IEEE Transactions on Instrumentation and Measurement, vol. 69, no. 6, pp. 3917-3927, Jun. 2020, doi: 10.1109/TIM.2019.2937447.

[27] D. Aguglia, P. Viarouge, and C. A. Martins, "Frequency-domain maximum-likelihood estimation of high-voltage pulse transformer model parameters," in IEEE Transactions on Industry Applications, vol. 49, no. 6, pp. 2552-2561, Nov. 2013, doi: 10.1109/TIA.2013.2265213.

\section{BIOGRAPHIES OF AUTHORS}

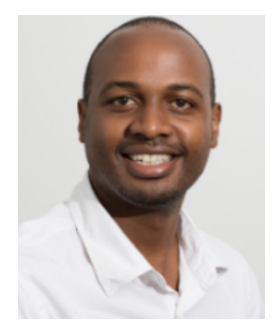

Fredrick Mukundi Mwaniki is a Lecturer with the department of Electrical and Electronic Engineering at the University of Stellenbosch. He received the B.Sc. degree in Electrical and Electronic Engineering from the University of Nairobi, Nairobi, Kenya, in 2009, and the B.Eng. and M.Eng. degrees in electrical engineering from the University of Pretoria, Pretoria, South Africa, in 2011 and 2013, respectively and the Ph.D. degree in electrical engineering from the Stellenbosch University in 2020. His researches are in fields of Energy Systems, Power Electronics, and Signal Processing. He is affiliated with IEEE as a member.

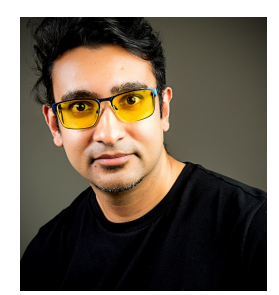

Ahmed Ali Sayyid is a Lecturer with the Department of Electrical and Information Engineering, at the University of Nairobi. He Recieved the BSc. degree in Electrical and Electronic Engineering from the University of Nairobi, Nairobi, Kenya in 2005 and B.Eng and M.Eng degrees in Electrical Engineering from the University of Pretoria, Pretoria, South Africa in 2011 and 2013 respectively. He has research interests in the fields of energy efficiency, renewable energy (solar-photovoltaic), power electronics, power systems and instrumentation. He is actively practising in analysis and engineering designs, among other consultancies for industry. He is affiliated with IET, AEE, and IEEE as a member. 